\title{
The Role of Dust in Producing the Cosmic Infrared Background
}

\author{
Eli Dwek \\ Laboratory for Astronomy and Solar Physics, NASA Goddard Space \\ Flight Center, Greenbelt, Maryland 20771, USA
}

\begin{abstract}
The extragalactic background light (EBL), exclusive of the cosmic microwave background, consists of the cumulative radiative output from all energy sources in the universe since the epoch of recombination. Most of this energy is released at ultraviolet and optical wavelengths. However, observations show that a significant fraction of the EBL falls in the 10 to $1000 \mu \mathrm{m}$ wavelength regime. This provides conclusive evidence that we live in a dusty universe, since only dust can efficiently absorbs a significant fraction of the background energy and reemit it at infrared wavelengths. The general role of dust in forming the cosmic infrared background (CIB) is therefore obvious. However, its role in determining the exact spectral shape of the CIB is quite complex. The CIB spectrum depends on the microscopic physical properties of the dust, its composition, abundance, and spatial distribution relative to the emitting sources, and its response to evolutionary processes that can modify all the factors listed above. This paper will present a brief summary of the many ways dust affects the intensity and spectral shape of the cosmic infrared background. In an Appendix we present new limits on the mid-infrared intensity of the CIB using $\mathrm{TeV} \gamma$-ray observations of Mrk 501.
\end{abstract}

\section{Introduction}

The extragalactic background light (EBL), exclusive of the cosmic microwave background, is the repository of all radiant energy releases in the universe since the epoch of recombination. Radiative sources contributing to the EBL include stars, which derive their energy from the nuclear processing of hydrogen into heavier elements, active galactic nuclei (AGN), which are powered by the release of gravitational energy associated with the accretion of matter onto a central black hole, and various exotic sources, such as decaying particles, primordial black holes, exploding stars, and substellar mass objects. Current limits on the $\mathrm{EBL}$, and the relative contribution of the various energy sources to the EBL are presented in these Symposium Proceedings by Hauser (2001) and in the review paper of Hauser \& Dwek (2001).

In a dust-free universe the EBL can, in principle, be simply derived from knowledge of the spectrum of the emitting sources and the cosmic history of their energy release. In a dusty universe the total intensity of the EBL is unchanged, but its energy is redistributed over the entire X-ray to far-infrared region of the 
spectrum. Predicting the EBL spectrum in a dusty universe therefore poses a significant challenge, since the exact frequency distribution of the reradiated emission depends on a large number of factors. On a microscopic level, the emitted spectrum depends on the wavelength dependence of the absorption and scattering properties of the dust, which in turn depend on the dust composition and size distribution. The reradiated spectrum also depends on the dust abundance and the relative spatial distribution of energy sources and absorbing dust. Finally, the cumulative spectrum from all sources depends on various evolutionary factors, including the history of dust formation and processes which destroy the dust, modify it, or re-distribute it relative to the radiant sources. Intergalactic dust, if present in sufficient quantities, can cause an overall dimming of the UV-optical output from distant sources, and produce a truly diffuse infrared background. In steady-state models for the universe, dust plays a more significant role, producing the cosmic microwave background via the thermalization of starlight by iron whiskers (Hoyle, Burbidge, \& Narlikar 1993). In the following I will examine in more detail the various factors and processes that determine the intensity and spectral energy distribution (SED) of the cosmic infrared background (CIB).

\section{Dust Properties}

The presence of dust in the interstellar medium (ISM) of the Milky Way is manifested in many different ways including the extinction, scattering, and polarization of starlight, the infrared emission, the interstellar depletion, and the presence of isotopic anomalies in meteorites. The most accepted interstellar dust model consists of a population of bare silicate and graphite grains with a power law distribution in grain radii extending from a few tens of angstroms to about $0.5 \mu \mathrm{m}$. An additional population of macromolecules, most commonly identified with polycyclic aromatic hydrocarbons (PAHs) must be added to this model. The very small dust particles and macromolecules are stochastically heated by the ambient radiation field, and give rise to the mid-infrared continuum spectrum and mid-infrared emission features observed in the Milky Way and in external galaxies.

Figure 1 (left panel) presents the mass absorption coefficient of graphite and silicate dust grains as a function of wavelength. The Figure illustrates the different absorption efficiencies of carbonaceous and silicate dust particles, the latter being significantly more transparent in the UV-visible regions of the spectrum. The efficiency of converting starlight to thermal infrared emission clearly depends on the relative abundance of carbonaceous-to-silicate dust in galaxies. Graphite particles posses a strong absorption feature at $2175 \AA$ seen in the extinction curve towards many stars in the Milky Way (MW) and nearby galaxies. The ratio of the $2175 \AA$-to-continuum extinction can be used to estimate the relative abundance of graphite-to-silicate dust in the interstellar medium towards the extincted source. The right panel of Figure 1 is a schematic representation of the observed average extinction in several local galaxies. The Figure shows an obvious trend of increasing strength of the $2175 \AA$ feature from the SMC, $\mathrm{LMC}$, the MW, and M51. The numbers next to each curve represent the average metallicity in these galaxies in solar units. The Figure suggests a trend of in- 

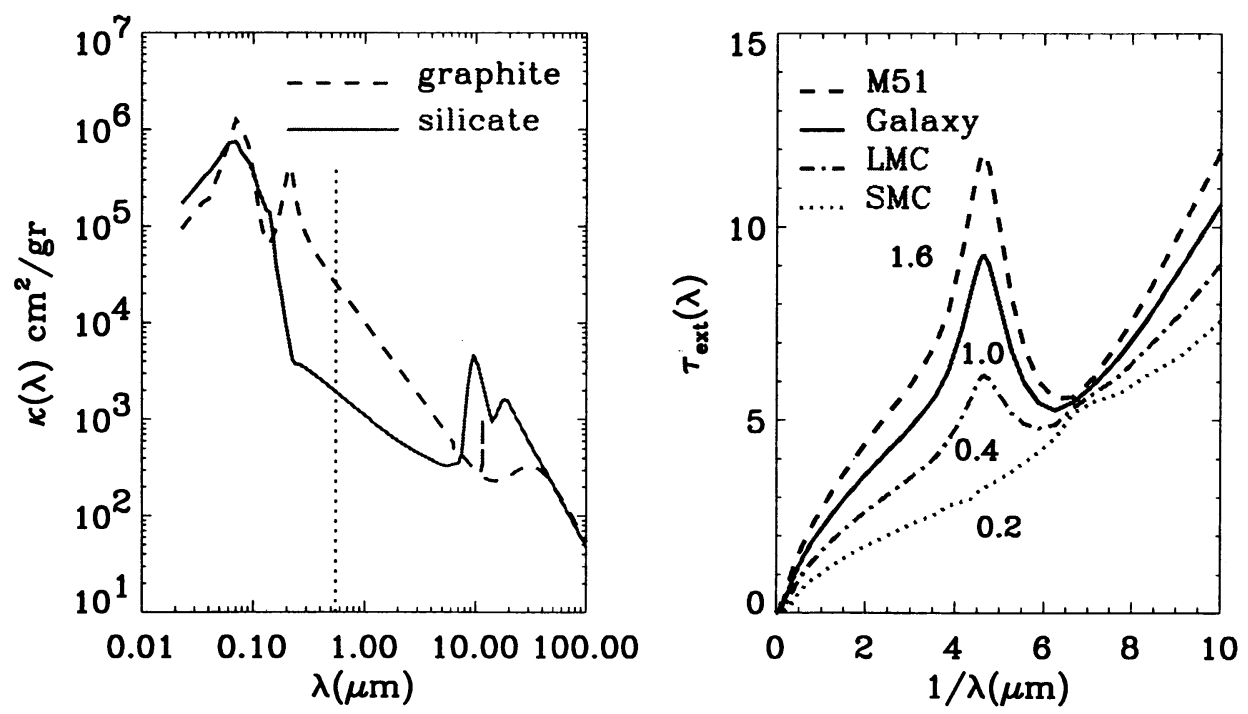

Figure 1. Left panel: the mass absorption coefficient of graphite and silicate grains. The dotted line gives the V-band position. Right panel: the average interstellar extinction in several local galaxies. Curves are labeled by the metallicity of the galaxies, normalized to that of the Milky Way.

creasing graphite-to-silicate dust ratio with metallicity. The trend may suggest that details of the galactic star formation history and stellar initial mass function play an important role in determining the dust composition and abundance, and hence the opacity, in galaxies.

\section{Relative Distribution of the Dust Compared to the Sources}

Given a dust abundance and composition, the most important parameter that determines the absorption efficiency of starlight and the spectrum of the reradiated infrared emission is the proximity of the dust to the radiation sources. One can distinguish between four different dust environments characterized by the spatial relation between the dust and the radiation sources: the circumstellar environment, consisting of dust that had recently formed out of the stellar ejecta and that is heated by the underlying stellar radiation field; the interstellar environment, consisting of dust residing in the ISM and heated by ionizing stars and/or the general interstellar radiation field; the AGN environment, consisting of a dusty torus, heated by the emission from the accretion disk; and the intergalactic environment, consisting of dust that has been expelled from galaxies and heated by the general diffuse background radiation.

\subsection{Circumstellar Dust}

Circumstellar dust dominates the mid-IR emission from intermediate age stellar populations (Bressan, Granato, \& Silva 1998), and may be an important 
source of thermal mid-IR emission in elliptical galaxies (Knapp, Gunn, \& WynnWilliams 1992). However, ISOCAM 4 and $15 \mu \mathrm{m}$ images of some elliptical galaxies reveal that the morphology of the $15 \mu \mathrm{m}$ dust emission component is significantly different from the $4 \mu \mathrm{m}$ stellar emission component (Madden, Vigroux, \& Sauvage 1999), suggesting an interstellar origin instead. Dust around young stellar objects will also have a very high efficiency for converting the radiation from the underlying object into infrared emission. However, the lifetime of this embedded phase is very short compared to the main sequence lifetime of these objects. Circumstellar dust therefore plays a minor role in the redistribution of energy in galaxies.

\subsection{Interstellar Dust and the Infrared Spectrum of Galaxies}

Most of the processing of galactic starlight is done by interstellar dust particles that reside in the different phases of the ISM. In fact, the infrared SEDs of all galaxies in the local universe can be constructed from a linear combination of several distinct emission components representing the different phases of the ISM: (1) a cirrus component, representing the emission from dust and carriers of the solid state infrared bands at $3.3,6.2,7.7,8.6,11.3$, and $12.7 \mu \mathrm{m}$, both residing in the diffuse atomic phase of the ISM and heated by the general interstellar radiation field; (2) a cold dust component, representing the emission from dust residing in molecular clouds, and heated by an attenuated interstellar radiation field; and (3) an $\mathrm{H}$ II or starburst emission component, representing the emission from dust residing in $\mathrm{H}$ II regions and heated by the ionizing radiation field. An additional AGN component may be needed to represent the spectra of some of the most luminous infrared galaxies. Using this simple procedure with two or more emission components, one can reproduce the fluxes and colors of IRAS galaxies with luminosities ranging from normal $\left(L \sim 10^{8.5} \mathrm{~L}_{\odot}\right)$ to the most luminous $\left(L \sim 10^{13} \mathrm{~L}_{\odot}\right)$ galaxies, and the observed trend of increasing $S(60 \mu \mathrm{m}) / S(100 \mu \mathrm{m})$ and decreasing $S(12 \mu \mathrm{m}) / S(25 \mu \mathrm{m})$ flux ratios with increasing infrared luminosity.

These empirical models offer a simple way of calculating the infrared spectra of galaxies in the local universe (Malkan \& Stecker 1998; Dwek et al. 1998; Guiderdoni et al. 1998; Rowan-Robinson \& Crawford 1989). However, to preserve the radiative energy balance of a galaxy, this emission must equal the amount of starlight absorbed by the dust. Calculating the opacity of galaxies poses a significant challenge, since in addition to the microscopic dust properties, the efficiency at which dust absorbs stellar photons depends on the dust abundance, the clumpiness of the ISM, and the relative distribution of stars and dust - all of which are evolving quantities.

Various radiative transfer models attempting to represent this complex reality were presented in the workshop on "The Opacity of Spiral Disks" (Davies $\&$ Burstein 1995). Models that include a 2-phase ISM consisting of molecular clouds and an intercloud component that calculate not only the attenuation of starlight but the re-radiated IR emission were developed, among others, by Silva et al. (1998), Városi \& Dwek (1999), and Misselt et al. (2000). The Városi \& Dwek model is analytic, and provides a very good approximation to the Monte-Carlo model of Witt \& Gordon (1996) that calculates the attenuation of radiation due to absorption and multiple scattering from clumpy spherical sys- 
tems. The models of Silva et al., which include a clumpy interstellar medium, are quite successful in fitting the UV to submillimeter wavelength emission of galaxy types ranging from ellipticals and spirals to starbursts and interacting systems. The observed SED of representative galaxies is characterized by an increasing $\mathrm{L}_{I R} / \mathrm{L}_{\text {opt }}$ ratio along the sequence from giant ellipticals, to spirals (NGC 6946), to starbursts (M 82), to mergers (Arp 220). Silva et al. attribute this trend to increasing infrared emission contributions from dust in giant starforming molecular clouds. In fact, the infrared spectrum of Arp 220 is almost identical to that of an H II region. These models provide a more physical approach to the construction of galaxy spectra, using radiative transfer methods to calculate the contribution of the various ISM dust components to the overall spectrum of the various types of galaxies.

\subsection{Dusty Torii Around Active Galactic Nuclei}

The energy output from AGN represents the radiative energy budget of the universe exclusive of the CMB and that released in nuclear burning processes. The energy of an AGN is derived from the release of gravitational energy associated with the accretion of matter onto a central black hole $(\mathrm{BH})$ located in the nucleus of a host galaxy. A significant fraction of this energy can be absorbed by the dusty torus around the central BH. The energy output from AGN-dominated galaxies is the sum of the reradiated thermal dust emission and the non-thermal synchrotron emission. The overall spectral energy distribution from these objects depends on the viewing angle. AGN that are viewed face-on have a synchrotron dominated power-law spectrum, whereas AGN that are viewed edge-on exhibit a thermal infrared excess consisting of a hot $(\sim 50$ $\mathrm{K}$ ) component commonly attributed to emission from the dusty torus, and a cooler $(\sim 20 \mathrm{~K})$ component, commonly attributed to reradiated stellar energy (Haas et al. 1998). In principle, the total bolometric contribution of AGN to the EBL could be comparable to the starlight contribution. However, direct observational correlation between $\mathrm{X}$-ray and IR/submillimeter sources suggests that AGN contribute only $\sim 10-20 \%$ of CIB intensity at 100 and $850 \mu \mathrm{m}$ (Barger et al. 2000). Their contribution at shorter wavelengths depends on the exact shape of the IR spectrum and is therefore still uncertain.

\subsection{Intergalactic Dust}

The progressive dimming of the light output of Type Ia supernovae with redshift has been taken as evidence that the mass density of the universe is subcritical, requiring a cosmological constant for closure (Perlmutter et al. 1999). An alternative scenario was suggested by Aguirre (1999) who argued that intergalactic dust could produce the same observational effect, alleviating the need to abandon the concept of a flat universe with a zero cosmological constant. Such intergalactic dust would be heated by the ambient intergalactic radiation field and produce a truly diffuse infrared background. Aguirre \& Haiman (2000) calculated the contribution of such dust to the CIB provided it was sufficiently abundant to account for the dimming of the distant supernovae. In particular, they found that intergalactic dust would produce most of the CIB at $850 \mu \mathrm{m}$. However, sources detected by the SCUBA survey account for over $50 \%$ of the CIB at this wavelength, leaving little room for any diffuse emission component. 


\section{The IR Evolution of Galaxies and Models for the CIB}

In order to calculate the contribution of galaxies to the CIB, one needs to know how their cumulative spectral energy density in the universe has evolved with time. Various models have been put forward to predict the CIB. These models can be grouped into four general categories: backward evolution, forward evolution, semi analytical, and cosmic chemical evolution models (see review by Hauser \& Dwek 2001). They differ in their degree of complexity, physical realism, and ability to account for various observational constraints or to make predictions. Backward evolution models are the simplest. They extrapolate the spectral properties and/or the comoving number density of local galaxies to higher redshifts using some parametric or unphysical form for their evolution. The main disadvantage of these models is that they are not constrained by the physical processes, such as star and metal formation, or radiative transfer processes that go on in the galaxies they represent.

Some of the shortcomings in backward evolution models are corrected in forward evolution models. At the heart of these models is a spectral evolution code which evolves the stellar populations and calculates the stellar, gas, and metallicity content and SED of a galaxy as a function of time. Initial conditions and model parameters are adjusted to reproduce the observational properties of galaxies in the local universe. Models for the diffuse interstellar dust emission vary in degree of complexity and physical input. As in the backward evolution models, the IR emission is represented by a sum of two or more components corresponding to the gas phases in which the dust resides and the radiation field it is exposed to. The various dust emission components are then evolved backwards in time in a manner that is determined by the evolution of the various physical parameters that determine their present intensity and spectral energy distribution.

Detailed spectral evolution models that follow the evolution of the dust composition and abundance, the galactic opacity, and the UV-to-far infrared spectral energy distribution for various stellar birthrate histories were constructed by Dwek (1998) and Dwek, Fioc, \& Városi (2000). The results of these calculations for spiral galaxies are shown in Figure 2.

The left panel of the Figure shows how the dust-to-metals as well as the carbon-to-silicate mass ratios evolve with time. The evolutionary trends reflect the temporal behavior of the evolution of the different stellar sources (carbon stars, $\mathrm{OH} / \mathrm{IR}$ stars, supernovae) that give rise to the dust composition. The right panel depicts the evolution of the V-band opacity perpendicular to the plane of the galaxy as a function of time. The Figure shows that a maximum opacity is reached at an epoch of $\sim 6$ Gyr. Figure 3 shows the various emission components contributing to the SED of a typical spiral galaxy at 12 Gyr (Fioc \& Dwek 2000). They include the infrared emission from H II regions and diffuse $\mathrm{H}$ I clouds. The contribution to the latter from PAH molecules, carbon and silicate dust is explicitly shown in the Figure.

Figure 4 examines the effect of the evolution of the dust composition and abundance on the SED of spiral galaxies, by plotting the spectral ratio of $\mathrm{S}_{\nu}(\mathrm{mw})$ to $S_{\nu}($ evol $)$, versus wavelength for various epochs. The spectrum $S_{\nu}(\mathrm{mw})$ is the SED calculated under the assumption that the dust-to-metal and the graphiteto-silicate mass ratios are constant, and equal to their currently observed Milky 

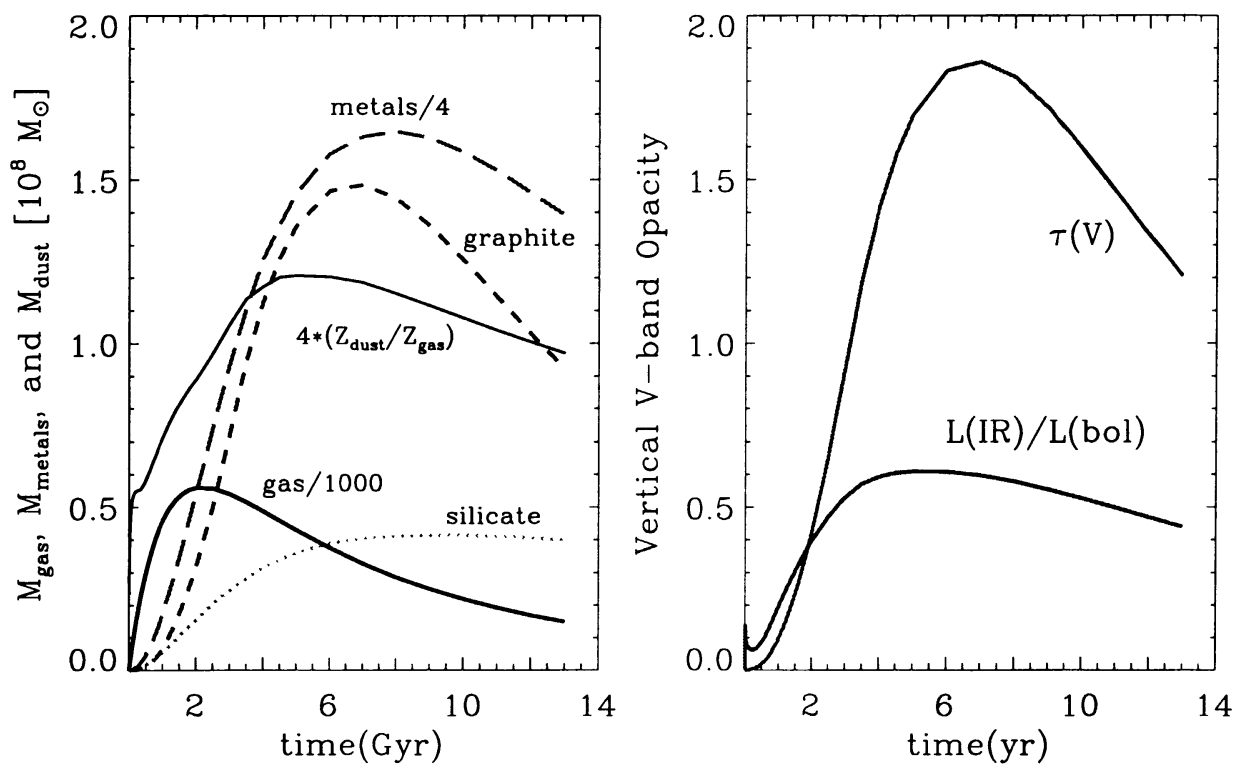

Figure 2. The evolution of various observable quantities in spiral galaxies as a function of time. See text for details.

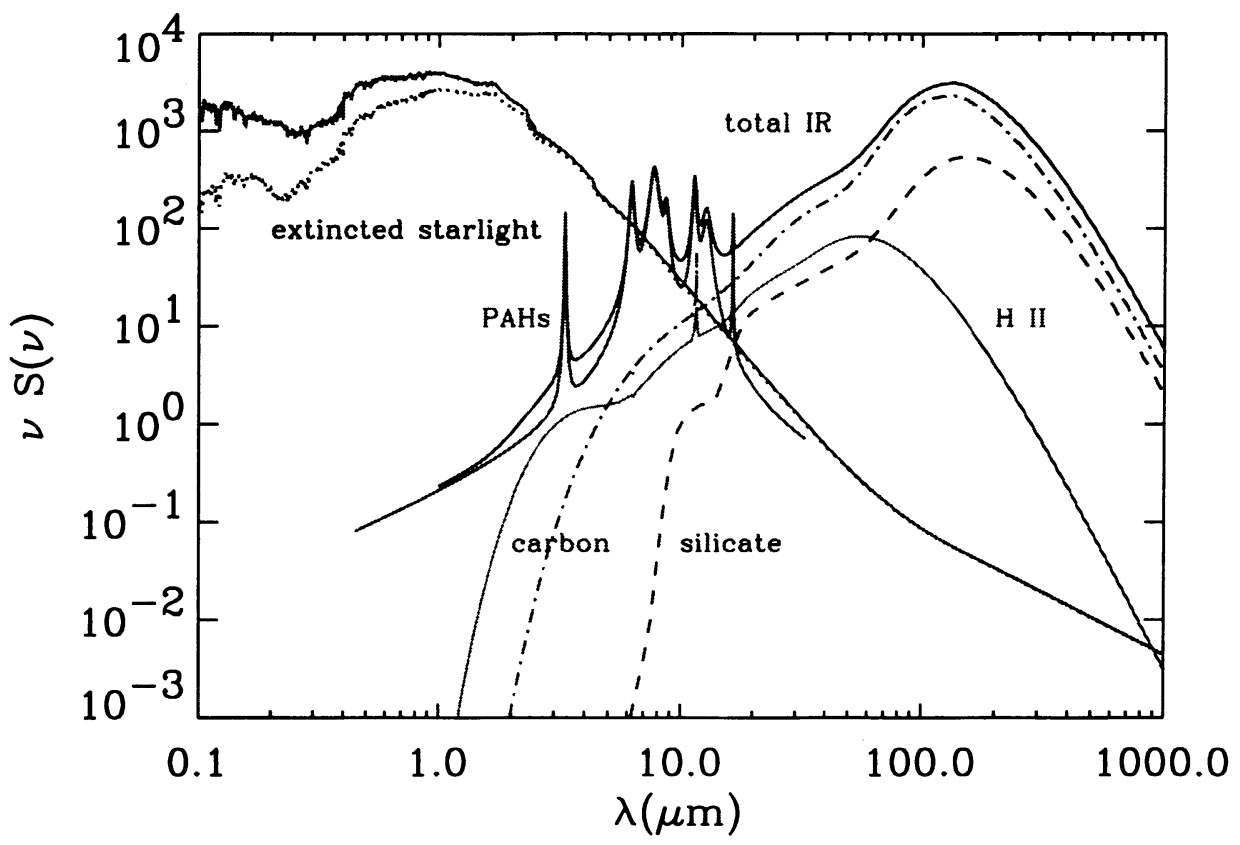

Figure 3. The UV to far-infrared spectrum of a spiral galaxy, and the various emission components contributing to the total spectrum. The top curve slanting down from upper left to lower right represents the unattenuated starlight from the galaxy. 


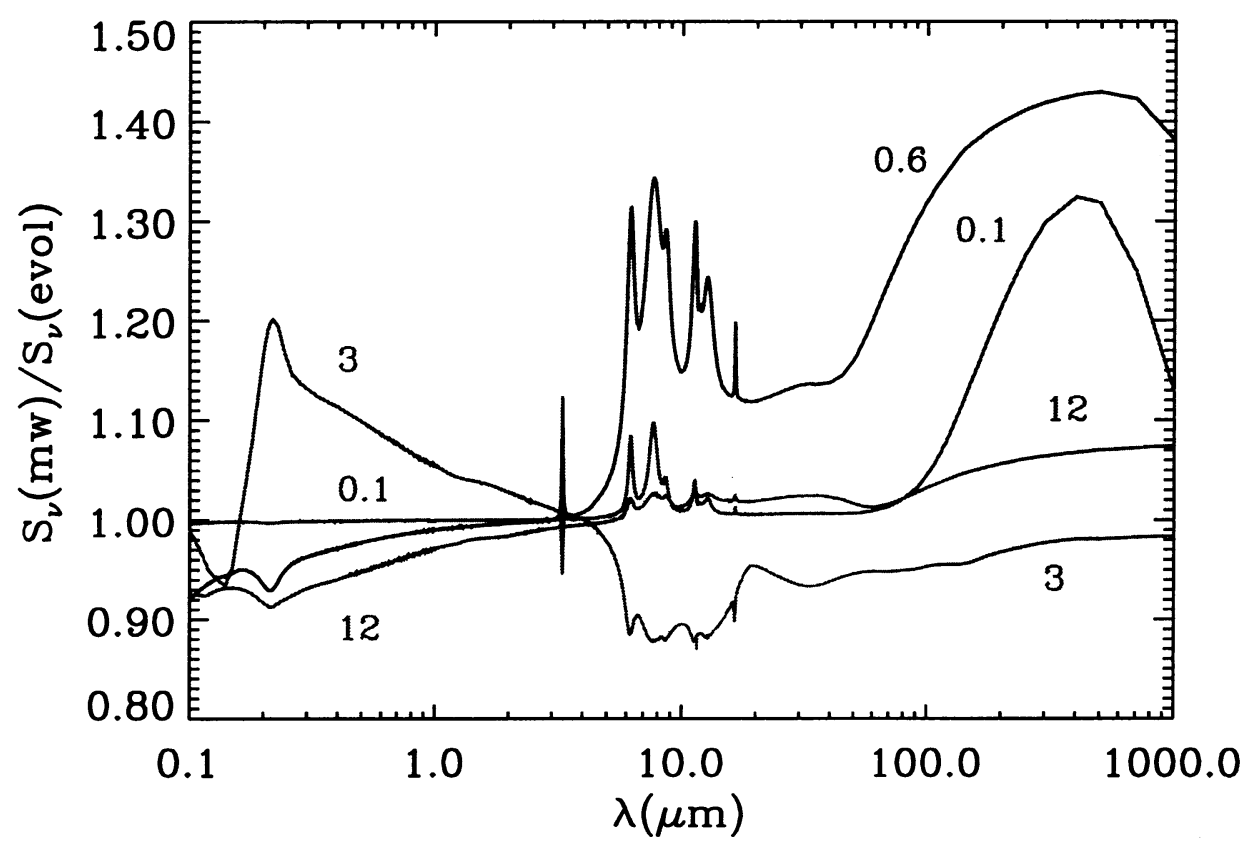

Figure 4. The spectral ratio $\mathrm{S}_{\nu}(\mathrm{mw}) / \mathrm{S}_{\nu}(\mathrm{evol})$ versus wavelength for various epochs in Gyr. See text for details.

Way values of 0.35 and 0.75 , respectively. The spectrum $S_{\nu}($ evol $)$ includes the detailed evolution of the metallicity dependent dust-to-gas and graphite-to-silicate dust mass ratios, and galactic opacity as depicted in Figures 2 and 3. The Figures show that non-evolving dust models over-predict the infrared emission at early epochs, especially the mid-infrared emission bands. Both models relax to the same spectrum at about $12 \mathrm{Gyr}$.

In forward evolution models galaxies evolve quiescently, with no allowance for any interaction or any stochastic changes in their star formation rate or morphology. In particular these models fail to match the SCUBA $850 \mu \mathrm{m}$ galaxy number counts without the ad hoc inclusion of a new population of ultraluminous infrared galaxies. The SCUBA observations of dust enshrouded galaxies at redshifts of $z \approx 2-3$, suggest a quick rise in galactic opacity. Figure 5 (based on the calculations of Dwek 1998; Városi \& Dwek 1999; and Dwek, Fioc, \& Városi 2000 ), shows the evolution of gas, metals, and dust mass in a pristine starburst (left panel) and the evolution of the attenuation of starlight (right panel) as a function of time. The Figure shows that starbursts can become effectively opaque in the UV and optical in a mere $100 \mathrm{Myr}$.

Most models for the CIB make similar predictions for the intensity and spectral energy distribution of the CIB, regardless of their degree of complexity or realism (Hauser \& Dwek 2001). This should not be surprising, since the CIB is the cumulative sum of energy outputs in the universe, and many details of the emission may be "washed out" in the summation process. The CIB is therefore 

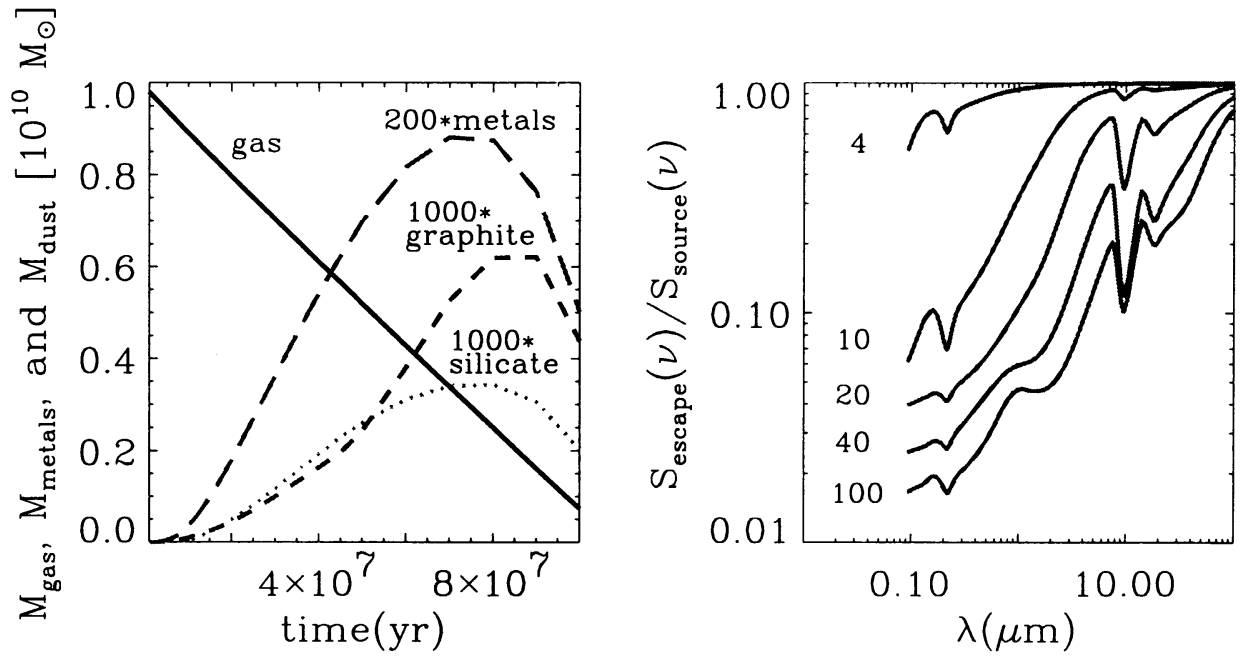

Figure 5. The evolution of various parameters (left panel) and the fraction of escaping radiation (right panel) from evolving pristine starbursts. The curves in the right panel are marked by the starburst's age in Myr.

not a strong discriminator between models, especially since it is still not well determined in the mid-infrared spectral region.

\section{Summary}

The very existence of the CIB provides conclusive evidence that we live in a dusty universe. The CIB is produced by the absorption and reemission of (mostly) starlight by interstellar dust in normal and active galaxies.

The CIB intensity and spectrum depend on a large number of microscopic and global parameters that affect the optical properties of the dust, its abundance and size distribution, the spatial distribution of the dust relative to the radiation sources, and the clumpiness of the interstellar medium in galaxies. Some physical processes, such as the stochastic heating of very small dust particles and macromolecules, may be responsible for a significant fraction of the CIB in the mid-infrared. In individual galaxies all these microscopic and global properties evolve with time. For example, it is very likely that the clumpiness in galaxies and the clump filling factor evolve in a fashion that depends on the total mass of gas in the galaxy, the supernova rate, and its metal content. Understanding the various physical processes that determine why local galaxies are the way they are, and how they evolved to their present conditions are crucial for predicting their spectral appearance at various redshifts.

The CIB represents, however, the time integrated emission from galaxies. There is therefore no unique way to determine the evolution of the various microscopic and global parameters from studying the CIB alone. 
Evolutionary models show that massive star forming regions rapidly become opaque at visible wavelengths. Consequently, the total energy released by these objects is mostly deposited in the CIB. The CIB can therefore provide a useful integral constraint on the star formation history of the universe. Different wavelength regions of the CIB sample the contribution of galaxies at different redshifts. Studies of the CIB can therefore provide important insights into the nature and evolution of the various sources contributing to the CIB.

Acknowledgments. I thank Mike Hauser for his comments and input on some sections of this paper, Ant Jones for his careful reading of and comments on the manuscript, and Rick Arendt for assistance in preparing the Appendix of the manuscript. This work was supported by the NASA Astrophysical Theory program OSS NRA 99-OSS-01.

\section{Appendix: Probing the Cosmic Infrared Background with $\mathrm{TeV} \gamma$-ray Sources}

Co-authored with Okkie C. de Jager

Potchefstroom University, Potchefstroom 2520, South Africa

The $\mathrm{TeV}$ spectrum of $\gamma$-ray sources can be attenuated by pair producing $\gamma-\gamma$ interactions with the extragalactic background light (EBL). Using recent detections of the EBL in the UV-optical (UVO) and far-infrared regions, we set limits on the cosmic infrared background (CIB) intensity in the $\sim 5$ to $60 \mu \mathrm{m}$ region with minimal assumptions on the intrinsic Mrk 501 source spectrum. The results are shown in the four panels of Figure 6. The upper left panel depicts the TeV spectra of Mrk 501 and Mrk 421. The panel to its right depicts the constraints used to construct possible EBL spectra. These include 3 possible UVO spectra, $100(5 \times 5 \times 4$ intensity combinations at 6,30 , and $60 \mu \mathrm{m})$, and a fixed far-IR spectrum, for a total of $300 \mathrm{EBL}$ spectra. The intrinsic Mrk 501 source spectra, corrected for attenuation by all EBL combinations, are shown in the lower left panel. Most source spectra exhibit an unphysical rise at > $10 \mathrm{TeV}$ energies. However, some spectra remain flat in $\mathrm{E}^{2} \mathrm{dN} / \mathrm{dE}$, as shown for select cases in the lower right panel. Two source spectra are shown for each choice of UVO spectrum. The upper curve depicts an unphysical spectral behavior, whereas the lower one corresponds to a $\mathrm{dN} / \mathrm{dE} \propto \mathrm{E}^{-2}$ power law. Preliminary upper limits on the CIB, derived by excluding EBL spectra giving rise to unphysical source spectra, are $5 \mathrm{nW} \mathrm{m}^{-2} \mathrm{sr}^{-1}$ in the $6-30 \mu \mathrm{m}$ interval and $<10 \mathrm{nW} \mathrm{m}^{-2} \mathrm{sr}^{-1}$ at $60 \mu \mathrm{m}$, for $\mathrm{H}_{0}=70 \mathrm{~km} \mathrm{~s}^{-1} \mathrm{Mpc}^{-1}$.

\section{References}

Aguirre, A. 1999, ApJ, 525, 583

Aguirre, A. \& Haiman, Z. 2000, ApJ, 532, 28

Barger, A. J., Cowie, L. L., Mushotzky, R. F., \& Richards, E. A. 2000, ApJ, submitted, astro-ph/0007175

Bressan, A., Granato, G. L., \& Silva L. 1998, A\&A, 332, 135 

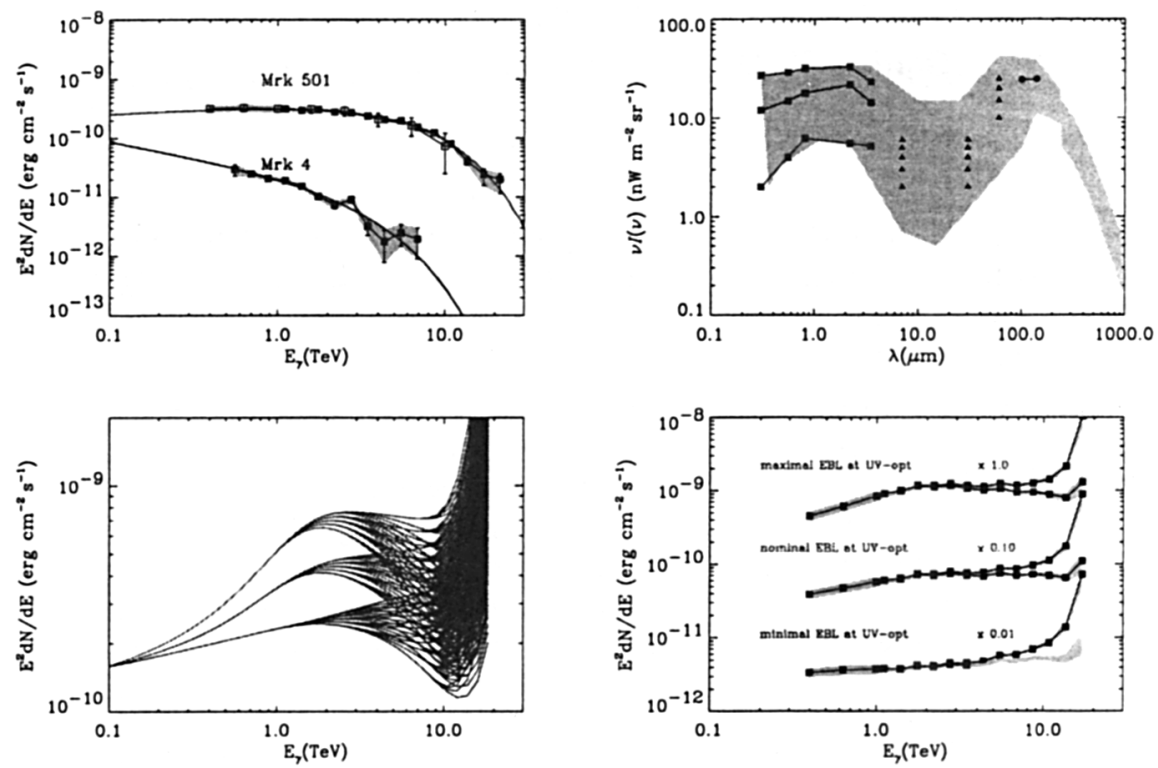

Figure 6. TeV spectra of Markarian 421 and 501, and EBL spectra (see text).

Davies, J. I., \& Burstein D. 1995, The Opacity of Spiral Disks (Dordrecht: Kluwer)

Dwek, E. 1998, ApJ, 501, 643

Dwek, E., et al. 1998, ApJ, 508, 106

Fioc, M., \& Dwek, E. 2000, in preparation

Dwek, E., Fioc, M., \& Városi, F. 2000, ISO Surveys of a Dusty Universe, ed. D. Lemke, M. Stickel, K Wilke (Springer: Berlin), 157

Guiderdoni, B., Hivon, E., Bouchet, F. R., \& Maffei, B. 1998, MNRAS, 295, 877 Haas, M, et al. 1998, ApJ, 503, L109

Hauser, M. G., \& Dwek, E. 2001, ARA\&A, 39, in press

Hauser, M. G. 2001, this volume

Hoyle, F., Burbidge, G., \& Narlikar, J. V. 1993, ApJ, 410, 437

Knapp, G. R., Gunn, J. E., \& Wynn-Williams, C. G. 1992, ApJ, 399, 76

Madden, S. C., Vigroux, L., \& Sauvage, M. 1999, The Universe as Seen by ISO, ed. P. Cox \& M. F. Kessler (Noordwijk: ESA Publication), 933

Malkan, M. A., \& Stecker, F. W. 1998, ApJ, 496, 13

Misselt, K. A., Gordon, K. D., Clayton, G. C., \& Wolff, M. J. 2000, ApJ, submitted

Perlmutter, S., et al. 1999, ApJ, 517, 565

Rowan-Robinson, M., \& Crawford, J. 1989, MNRAS, 238, 523

Silva, L., Granato, G. L., Bressan, A., \& Danese, L. 1998, ApJ, 509, 103 
Városi, F., \& Dwek, E. 1999, ApJ, 523, 265

Witt, A. N., \& Gordon, K. D. 1996, ApJ, 463, 681

\section{Discussion}

Mike Hauser: The shaded region in your figure comparing models with CIB measurements is the same as the $\pm 2 \sigma$ region in my talk on the observations of the CIB, earlier in this Symposium.

Eli Dwek: Right. 